\title{
Evolutionary and genetic features of drug targets
}

\author{
Y. Quan ${ }^{1}$, Z.Y. Wang ${ }^{1,2}$, X.Y. Chu ${ }^{1}$, H.Y. Zhang ${ }^{1 *}$ \\ ${ }^{1}$ Hubei Key Laboratory of Agricultural Bioinformatics, College of Informatics, Huazhong Agricultural \\ University, Wuhan, P. R. China \\ ${ }^{2}$ Center for Molecular Biology of the University of Heidelberg (ZMBH), Heidelberg, Germany \\ *e-mail: zhy630@mail.hzau.edu.cn
}

Key words: drug discovery, drug target, genetics, evolutionary biology

Motivation and Aim: In the modern drug discovery pipeline, identification of novel drug targets is a critical step. Despite rapid progress in developing biomedical techniques, it is still a great challenge to find promising new targets from the ample space of human genes. This fact is partially responsible for the situation of "more investments, fewer drugs" in the pharmaceutical industry. To escape this predicament, novel concepts should be introduced in this field.

A series of recent researches revealed that successfully targeted genes share some common evolutionary and genetic features [1-3], which means that the knowledge accumulated in modern evolutionary biology and genetics is very helpful to identify potential drug targets and to find new drugs as well. In this article, we comprehensively summarize the links between human drug targets and genetic diseases and their evolutionary origins, with an attempt to introduce these novel concepts and their medical implications to the biomedical community.

Results: Genetics-derived disease genes, especially those strongly linked with disease phenotypes, are a rich source of drug targets. Besides, the genetic knowledge about the pathogenesis, that is, whether the diseases arise from loss-of-function (LOF) or gain-offunction (GOF) mutations of the responsible genes, provides critical information about drug mode of action. More interestingly, the evolutionary background of human genes is also valuable in drug target identification. The genes originated in certain evolutionary stages are more druggable and ohnolog genes have higher probability to serve as drug targets, because of their sensitivity to dosage variance.

Conclusion: It seems that during the paradigm shift of pharmaceutical industry in this omics era, modern genetics and evolutionary biology will play a more and more important role.

Acknowledgements: Supported by the Fundamental Research Funds for the Central Universities (Grants No. 2662015PY004 and No. 2662017PY115).

\section{References}

1. Wang Z.Y., Fu L.Y., Zhang H.Y. (2012) Can medical genetics and evolutionary biology inspire drug target identification? Trends Mol. Med. 18(2):69-71.

2. Sun H.Y., Hou T.J., Zhang H.Y. (2014) Finding chemical drugs for genetic diseases. Drug Discov. Today. 19(12):1836-1840.

3. Kamb A., Harper S., Stefansson K. (2013) Human genetics as a foundation for innovative drug development. Nat. Biotechnol. 31(11):975-978. 